\title{
Microbiology Specimen Planned Time Point Number
}

National Cancer Institute

\section{Source}

National Cancer Institute. Microbiology Specimen Planned Time Point Number. NCI

Thesaurus. Code C87923.

The numerical identifier of a microbiology specimen point in time. 